\title{
Prevalence of shigellosis diarrhoea in a paediatrics population: hospital based survey in Bangui, Central African Republic
}

\author{
Alexandre Manirakiza ${ }^{1}$, Prisca Megne-Boudjeka², Gustave Bobossi-Serengbe ${ }^{2,3}$, Raymond Bercion ${ }^{4}$, \\ Alain Le Faou ${ }^{5}$ \\ ${ }^{1}$ Institute Pasteur of Bangui, Bangui, Central African Republic \\ ${ }^{2}$ Complexe Pediatrique de Bangui, Bangui, Central African Republic \\ ${ }^{3}$ University of Bangui, Bangui, Central African Republic \\ ${ }^{4}$ Federation of Laboratories, Hôpital Principal, Dakar, Senegal \\ ${ }^{5}$ Laboratoire de Virologie, CHU de Nancy, Hopital de Brabois adultes, Cedex, France
}

\begin{abstract}
Introduction: Shigellosis is still a major public health problem in sub-Saharan countries, especially among children.

Methodology: The prevalence of shigellosis in children presenting with diarrhoea in the Complexe Pédiatrique de Bangui, Central African Republic, was determined. Stools were analyzed in the bacteriology laboratory of the Institut Pasteur de Bangui, Central African Republic, where identification of Shigella species and analysis of antibiotics susceptibility were performed.

Results: A total of 15 strains of Shigella were isolated from 156 stools; Shigella flexneri was the only species found. Two infected children died of dehydration. Most strains were resistant to antibiotics except quinolones, which were active on all of these strains.

Conclusions: The control of Shigella infections should be reinforced in Bangui, and accurate, affordable and rapid methods of diagnosis would be helpful.
\end{abstract}

Key words: shigellosis; paediatrics; Central Africa

J Infect Dev Ctries 2010; 4(10):655- 657.

(Received 16 November 2009 - Accepted 10 June 2010)

Copyright (c) 2010 Manirakiza et al. This is an open-access article distributed under the Creative Commons Attribution License, which permits unrestricted use, distribution, and reproduction in any medium, provided the original work is properly cited.

\section{Introduction}

Diarrhoea is a major cause of mortality and morbidity in developing countries [1]. Shigellosis is a significant cause, especially in children under five years old [2]. Shigella flexneri is the leading cause of shigellosis, particularly in the poor populations [3]. Shigellosis is endemic in Central African Republic (CAR), where an outbreak of Shigella dysenteriae type 1 was recently reported [4]. The present study was undertaken to determine the prevalence and the antimicrobial susceptibility patterns of Shigella serotypes in children hospitalized for diarrhoea in a paediatric unit in Bangui, CAR.

\section{Methodology}

Study population

This study was undertaken to evaluate the prevalence of in diarrhoeic stools at the Complexe Pédiatrique of Bangui, which is the only children's hospital in the city. From March to August 2007, all children aged between one and 59 months who had acute diarrhoea (at least three stool passages per day) for less than five days and who did not receive any antibiotics were included in this study after parents or a tutor gave formal consent. The parent or tutor was asked about the characteristics of the stools: mucoid, and/or watery, and/or bloody. These observations were noted macroscopically by the investigator when the stool samples were collected for laboratory analysis. A total of 156 children from Bangui city and its periphery were included, of whom 84 were male (sex-ratio $=1.2$ ). The mean age was 12.4 months, and $57 \%$ of the children were between one and 11 months.

Fresh stool specimens were collected in a sterile container and brought within one hour to the laboratory of the Institut Pasteur de Bangui where a rapid test for qualitative detection of rotavirus and adenovirus (VIKIA Rota-Adeno, BioMerieux, Marcy l'Etoile, France) was performed before bacteriology. 


\section{Bacteriology}

Systematic Gram coloration was performed on all samples. During this analysis, microscopic bloody stools were noted as well as the Gram characteristics for bacterial positive samples. The stools samples were then inoculated immediately with Hektoen, (BioMérieux), Bromocresol Purple (Bio-Mérieux), and Mac Conkey sorbitol (Becton Dickinson, Sparks, MD, USA) agar, and on selenite media (Bio-Rad, Marnes-la-Coquette, France) for enrichment. Nonlactose-fermenting strains were systematically tested for their urease activity and for their manitol motility. Shigella strains were identified with API 20E strips (BioMérieux) and agglutinated with sera against Shigella dysenteriae, Shigella flexneri, Shigella boydii and Shigella sonnei (Bio-Rad).

Antimicrobial susceptibility testing was done by disk diffusion on Mueller-Hinton agar as recommended by the Antibiogram Committee of the French Society for Microbiology (CA-SFM) [5]. The following antimicrobials (Bio-Rad) were tested: amoxicillin, amoxicillin-clavulanic acid, ticarcillin, cefoxitin, cefotaxime, cephalotin, chloramphenicol, tetracycline, gentamicin, nalidixic acid, ciprofloxacin, streptomycin, sulphonamides and sulphamethoxazole-trimethoprim. Diameters were measured with the automated Osiris system (BioRad). Bacteria were classified as susceptible, intermediate, or resistant, according to the CA-SFM interpretation of results.

\section{Data analysis}

Data were entered and analyzed using Epi Info 3.3.2 software (Centers for Disease Control and prevention, Atlanta, USA).

\section{Results}

Stools were watery $(67.3 \%)$, mucoid $(25.6 \%)$ or bloody (7.1\%). Shigella strains were isolated from 15 samples $(9.6 \%)$ and all identified as Shigella flexneri. A total of $11(73.3 \%)$ of the samples were from female children. Cases were distributed independently of the social and economic status of their families. Two of the infected children died of dehydration. Seven stools contained Salmonella enterica. Rotavirus and adenovirus were found respectively in 14 and 7 stools, which tested negative for Salmonella and Shigella.

Most strains of Shigella flexneri were resistant to amoxicillin, chloramphenicol, cotrimoxazole tetracycline and streptomycin; nine different patterns of resistance were observed. All the strains remained susceptible to nalidixic acid and ciprofloxacin. Details are shown in Table 1 and Figure 1.

Table 1. Resistance profiles of Shigella flexneri isolated at the 'Complexe Pédiatrique' of Bangui, CAR

\begin{tabular}{|l|l|}
\hline Resistances & Frequency \\
\hline AMX S C & 1 \\
\hline AMX S C Te & 1 \\
\hline AMX S C SXT Te & 1 \\
\hline AMX S C SXT & 1 \\
\hline AMX SXT Te & 1 \\
\hline AMX AMC CF S C SXT Te & 1 \\
\hline S SXT Te & 2 \\
\hline AMX S C SXT Te & 3 \\
\hline AMX AMC S C SXT Te & 4 \\
\hline Total & 15 \\
\hline
\end{tabular}

AMX : amoxicillin ; AMC : amoxicillin-clavulanic acid ; S : streptomycine

$\mathrm{C}$ : chloramphenicol; SXT : cotrimoxazole; Te : tetracycline; CF : cefalotine

Figure 1. Susceptibility of Shigella flexineri to antibiotics in Bangui, CAR

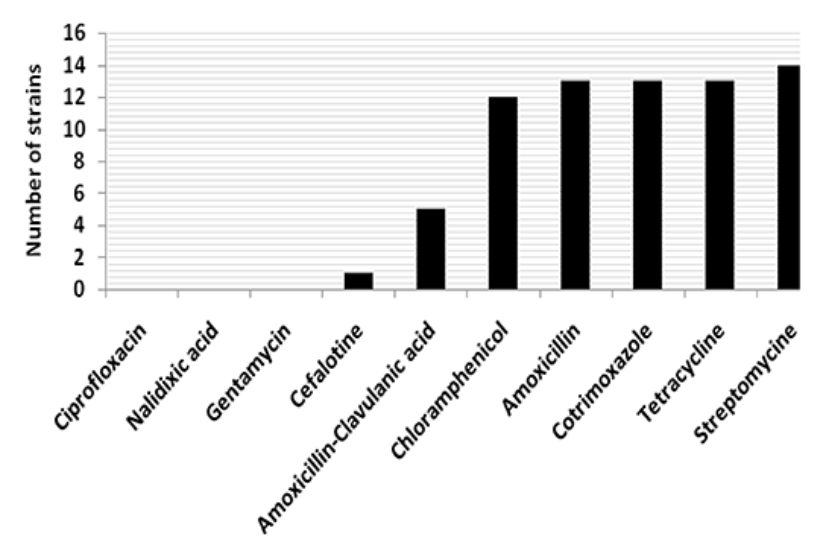

\section{Discussion}

Children under five years of age are a high-risk group for diarrhoea. Shigella is a major cause, as reported in Senegal, Djibouti and Zambia, where it represents respectively $11 \%, 15 \%$ and $10.2 \%$ of enteropathogenic agents [6-8]. A previous study in Bangui in 1983 found only 2.2\% of diarrhoea attributable to Shigella (9). Such an increase in prevalence is supported by World Health Organization data, which demonstrates a regular 
increasing trend of Shigella in sub-Saharan countries in the last ten years [10].

In 2003, 2004 and 2005, Shigella dysenteriae was responsible for two epidemic episodes in CAR [4, 11], but in our hospital survey, Shigella flexineri was the only species isolated. Thus, Shigella flexineri is apparently endemic to the population as described in Tunisia, while Shigella dysenteriae would be mainly associated with outbreaks [3].

The management of shigellosis is a problem due to its acquired resistance to commonly used antibiotics, which is increasing. Fortunately, ciprofloxacin has retained its efficiency, although it is given orally, which may create a problem in seriously ill children. Nevertheless, as recently reported by Bercion and collaborators in 2008, the full sensitivity of Shigella to quinolones is an unexpected advantage for practitioners in the treatment of shigellosis in CAR. However, this advantage is not common elsewhere in Africa[13].

It has been reported that shigellosis prevalence is extensively underestimated because Shigella are very fragile and sometimes in low number in faeces, which necessitates inoculation of media within four hours after stool emission and the use of enrichment medium [14]. In the current study, this disadvantage was taken into account. The authors minimized the risk of missing a diagnosis of shigellosis by a rapid inoculation of media, which was facilitated by the vicinity of the two institutions. It should also be noted that Shigella is the most prevalent pathogen found, which justifies improving the diagnosis of shigellosis in everyday practice. Although molecular techniques are rapid and sensitive in the diagnosis of Shigella infection, they cannot be put into practice on a routine basis. Shigella remains a burden in developing countries; sensitization of the population and practitioners to this lethal infection $(2 / 15$ in the current study) would contribute to limiting its severity and prevalence.

\section{Acknowledgements}

We are grateful to all the parents and tutors who consented to participating in this study. We also thank the staff of the Complexe Pédiatrique de Bangui, and especially Dr. Jean Chrysostome Gody, who kindly collaborated in data collection.

\section{References}

1. O'Ryan (2005) A millennium Update on Pediatric diarrheal illness in the developing world. Semin Pediatr Infect Dis 16: 125-136.

2. Navia MM, Capitano L, Ruiz J, Vargas M, Urassa H, Schellemberg D, Gasco J and Vila J (1999) Typing and characterization of mechanisms of resistance of Shigella spp. isolated from feces of children under 5 years of age from Ifakara, Tanzania. J Clin Microbiol 37: 3113-3117.

3. Naik DG (2006) Prevalence and antimicrobial susceptibility patterns of Shigella species in Asmara, Eritrea, northeast Africa. J Microbiol Immunol Infect 39: 392-395.

4. Bercion R, Demartin M, Recio C, Massamba PM, Frank T, Escriba JM, Grimont PA and Weill FX (2006) Molecular epidemiology of multidrug-resistant Shigella dysenteriae type 1 causing dysentery outbreaks in Central African Republic, 2003-2004. Trans R Soc Trop Med Hyg 100: 1151-1158.

5. Comité de l'Antibiogramme de la Société Française de Microbiologie (CA-SFM). Communiqué 2005, Paris, France: http://www.sfm.asso.fr.

6. Bercion R, Martin D, Toussaint P, Graffin B and Windeck I (1994) Epidémiologie et bactériologie des shigelloses à Djibouti. A propos de 555 souches de Shigella isolées en 21 mois. Med. Mal Infect 24: 622-627.

7. Gassama A, Boye CS, Adiara A, Raphenon G, Sam A and Mboup S (1997) Shigella au Sénégal (1993-1994): distribution par sérovar, sensibilité aux antibiotiques et virulence. Med Mal Infect 27: 267-270.

8. Nakano $T$, Kamiya $H$, Matsubayashi N, Watanabe M, Sakurai M, Honda T (1998) Diagnosis of bacterial enteric infections in children in Zambia. Acta Paediatr Jpn 40: 259263.

9. Georges MC, Roure C, Tauxe RV, Meunier DM, Merlin M, Testa J, Baya C, Limbassa J and Georges AJ (1987) Diarrheal morbidity and mortality in children in the Central African Republic. Am J Trop Med Hyg 36: 598-602.

10. Organisation Mondiale de la Santé (2005) Shigellose: charge de morbidité, épidémiologie et prise en charge des cas. Rev Epidémiol Hebdomadaire 80: 94-99.

11. Bonn D (2005) Infectious disease surveillance update Lancet Infect Dis 5: 540.

12. Bercion R, Njuimo SP, Boudjeka PM, Manirakiza A (2008) Distribution and antibiotic susceptibility of Shigella isolates in Bangui, Central African Republic. Trop Med Int Health 13: 468-471.

13. Tiruneh M (2009) Serodiversity and antimicrobial resistance pattern of Shigella isolates at Gondar University teaching hospital, Northwest Ethiopia. Jpn J Infect Dis 62: 93-97.

14. LeMinor L and Richard C. Méthodes de laboratoire pour l'identification des entérobactéries. Institut Pasteur de Paris 1993.

\section{Corresponding author}

Dr. Alexandre Manirakiza

Institut Pasteur de Bangui

Avenue Pasteur, PO box 923, Bangui, Central African Republic

Phone: (236)70 930579

Fax: (236) 61210109

Email: amanirak@yahoo.fr

Conflict of interests: No conflict of interests is declared. 